\title{
Discovery of two novel and adjacent QTLs on chromosome B02 controlling resistance against bacterial wilt in peanut variety Zhonghua 6
}

\author{
Huaiyong Luo ${ }^{1} \cdot$ Manish K. Pandey ${ }^{2} \cdot \mathrm{Ye} \mathrm{Zhi}^{3} \cdot$ Huan Zhang $^{1} \cdot$ Siliang Xu${ }^{1} \cdot$ Jianbin Guo ${ }^{1} \cdot$ Bei Wu ${ }^{1} \cdot$ Haiwen Chen $^{1}$. \\ Xiaoping Ren ${ }^{1} \cdot$ Xiaojing Zhou $^{1}$ - Yuning Chen ${ }^{1} \cdot$ Weigang Chen ${ }^{1} \cdot$ Li Huang $^{1} \cdot$ Nian Liu $^{1} \cdot$ Hari K. Sudini ${ }^{2}$. \\ Rajeev K. Varshney ${ }^{2}$ ' Yong Lei ${ }^{1} \cdot$ Boshou Liao $^{1} \cdot$ Huifang Jiang ${ }^{1}$ (D)
}

Received: 7 October 2019 / Accepted: 3 January 2020 / Published online: 24 January 2020

(c) The Author(s) 2020

\begin{abstract}
Key message Two novel and adjacent genomics and candidate genes for bacterial wilt resistance were identified on chromosome B02 in peanut variety Zhonghua 6 using both traditional QTL mapping and QTL-seq methods.

Abstract Peanut (Arachis hypogaea) is an important oilseed crop worldwide. Utilization of genetic resistance is the most economic and effective approach to control bacterial wilt, one of the most devastating plant diseases, in peanut production. To accelerate the genetic improvement of bacterial wilt resistance (BWR) in peanut breeding programs, quantitative trait locus (QTL) mapping has been conducted for two resistant varieties. In this context, we deployed linkage mapping as well as sequencing-based mapping approach, QTL-seq, to identify genomic regions and candidate genes for BWR in another highly resistant variety Zhonghua 6 . The recombination inbred line population ( 268 progenies) from the cross Xuhua $13 \times$ Zhonghua 6 was used in BWR evaluation across five environments. QTL mapping using both SSR- and SNP-based genetic maps identified a stable QTL ( $q$ BWRB02-1) on chromosome B02 with 37.79-78.86\% phenotypic variation explained (PVE) across five environments. The QTL-seq facilitated further dissection of $q B W R B 02-1$ into two adjacent genomic regions, $q B W R B 02-$ 1-1 (2.81-4.24 Mb) and $q B W R B 02-1-2(6.54-8.75 \mathrm{Mb})$. Mapping of newly developed Kompetitive allele-specific PCR (KASP) markers on the genetic map confirmed their stable expressions across five environments. The effects of $q B W R B 02-$ 1-1 (49.43-68.86\% PVE) were much higher than qBWRB02-1-2 (3.96-6.48\% PVE) and other previously reported QTLs. Nineteen putative candidate genes affected by 49 non-synonymous SNPs were identified for $q B W R B 02-1-1$, and ten of them were predicted to code for disease resistance proteins. The major and stable QTL $q B W R B 02-1-1$ and validated KASP markers could be deployed in genomics-assisted breeding (GAB) to develop improved peanut varieties with enhanced BWR.
\end{abstract}

Communicated by Albrecht E. Melchinger.

Electronic supplementary material The online version of this article (https://doi.org/10.1007/s00122-020-03537-9) contains supplementary material, which is available to authorized users.

Boshou Liao

lboshou@hotmail.com

Huifang Jiang

peanutlab@oilcrops.cn

1 Key Laboratory of Biology and Genetic Improvement of Oil Crops, Ministry of Agriculture, Oil Crops Research Institute of the Chinese Academy of Agricultural Sciences (CAAS), Wuhan 430062, China

2 International Crops Research Institute for the Semi-Arid Tropics (ICRISAT), Hyderabad 502324, India

3 Angel Yeast Co., Ltd, Yichang 443003, Hubei, China

\section{Introduction}

The cultivated peanut (Arachis hypogaea L.), also known as groundnut, is an important legume crop grown in more than 100 countries with 47.10 million tonnes global production in 2017 (FAOSTAT 2019). It is consumed worldwide in the form of oil, nuts, peanut butter, candy, etc. (Varshney et al. 2013). Bacterial wilt disease poses a serious threat to peanut production in China, the highest peanut producer in the world, in addition to Vietnam, Indonesia, Uganda and other Southeast Asian countries. This disease usually causes $10-30 \%$ yield losses and can cause up to $50-100 \%$ in severe circumstances (Jiang et al. 2017). It is caused by the soilborne bacterium Ralstonia solanacearum which has a wide host range including over 450 plant species belonging to 
more than 50 botanical families (Deslandes and Genin 2014; Salanoubat et al. 2002).

Deploying host resistance is the most economical and eco-friendly approach to control bacterial wilt disease in peanut production. To do so, it is very important to identify stable resistance sources with high survival rates under heavy bacterial wilt disease pressure based on multi-environment disease screening (Mallikarjuna and Varshney 2014). More than 140 peanut landraces, improved varieties or wild Arachis species were identified to possess high levels of resistance to bacterial wilt (Liao 2017). Cultivation of resistant varieties has greatly reduced yield loss in peanut production to less than 5\%. However, the breeding of new resistant varieties faces challenges due to the inability of conducting large-scale screening in conventional breeding programs (Jiang et al. 2017). Under such circumstances, it is essential to identify QTLs and linked markers for bacterial wilt resistance (BWR), which could be deployed in genomics-assisted breeding (GAB) to accelerate the breeding process (Janila et al. 2016; Varshney et al. 2014).

Up to now, QTL mapping has been conducted for only two resistant varieties, i.e., Yueyou 92 and Yuanza 9102, through either traditional genetic map-based QTL mapping or sequencing-based mapping approaches. Based on a $1627.4 \mathrm{cM}$ genetic map containing 237 SSR and SNP markers, Zhao et al. (2016) identified a major QTL $q B W-1$ for BWR in an $\mathrm{F}_{2}$ population and its recombination inbred line (RIL) population derived from the crossing of Yueyou 92 and susceptible variety Xinhuixiaoli. The resistance in Yueyou 92 is from a Chinese landrace Xiekangqing, which is a major resistance source utilized for bacterial wilt disease in Chinese breeding programs (Mallikarjuna and Varshney 2014). In addition to traditional genetic map-based QTL mapping, sequencing-based mapping approaches, such as QTL-seq (Takagi et al. 2013), facilitate high resolution and faster mapping of genomic regions and candidate genes for target traits in crop plants (Pandey et al. 2016; Wambugu et al. 2018; Zhong et al. 2018). Based on the genome sequences of $A$. duranensis and A. ipaensis (the diploid ancestors of allotetraploid cultivated peanut) (Bertioli et al. 2016), the QTL-seq approach was deployed for the first time in a RIL population and helped in rapid discovery of the major and stable QTL $q B W R B 02.1$ for BWR in Yuanza 9102 as well as its candidate genes and diagnostic markers (Luo et al. 2019). The resistance source for Yuanza 9102 was suggested to be a resistant wild species A. diogoi (Mallikarjuna and Varshney 2014). More importantly, the resistant allele of $q B W R B 02.1$ was absent from other resistant peanut genotypes according to diagnostic markers genotyping (Luo et al. 2019). Therefore, more efforts are needed to discover the QTLs for BWR in other resistant genotypes using either traditional genetic map-based QTL mapping or sequencing-based mapping approaches, which would increase the diversity of utilizable resistant QTLs/ genes in peanut breeding and might improve the resistance level by integrating multiple resistance alleles.

The improved peanut variety Zhonghua 6 has been highly and stably resistant to bacterial wilt disease since released by the Oil Crops Research Institute of the Chinese Academy of Agricultural Sciences in 2000. Its resistance derives from the Chinese landrace Taishan Zhenzhu, which is highly resistant but seldom used in peanut breeding because of undesirable features such as small-seeded and low-yield. Compared to Taishan Zhenzhu, Zhonghua 6 has improved agronomic traits thus would be an important resistance source in future breeding programs. In the present study, a recombination inbred line (RIL) population (268 progenies) developed from the cross of Xuhua 13 (highly susceptible) $\times$ Zhonghua 6 was used to discover the genetic basis of BWR in Zhonghua 6 . More SSR loci were added to the previously published SSRbased genetic map (Luo et al. 2018) in the present study, and QTL mapping were conducted with phenotyping data across five environments and SSR- and SNP-based genetic map. In addition, based on the recently published genome sequence of cultivated peanut (Chen et al. 2019), the QTLseq approach was deployed as well to identify the genomic regions, candidate genes and efficient markers for BWR in the present study.

\section{Materials and methods}

\section{Plant materials}

The improved peanut variety Xuhua 13 is highly susceptible to bacterial wilt disease, while Zhonghua 6 has been highly and stably resistant since it was released. A RIL population (268 lines) was developed from the cross Xuhua $13 \times$ Zhonghua 6 following single seed decent (SSD) method (Luo et al. 2018). Our previous study reported SSR-based genetic mapping using a subset (187 RILs) of the mapping population leading to development of a genetic map with 817 SSR loci (Luo et al. 2018). In the present study, DNA was extracted from the same subset in the $\mathrm{F}_{5}$ generation for genotyping of SSR markers to improve the genetic map. Recently, 186 of the 187 RILs in the $\mathrm{F}_{6}$ generation were genotyped by ddRADseq (double-digest Restriction-site Associated DNA sequencing), upon which a SNP-based genetic map was constructed with 2595 SNPs, spanning $2465.62 \mathrm{cM}$ with an average inter-marker distance of $0.95 \mathrm{cM}$ (Liu et al. 2019). Three generations $\left(\mathrm{F}_{6}-\mathrm{F}_{8}\right)$ of the RIL population (268 lines) were used for phenotyping BWR. The $\mathrm{F}_{9}$ generation of the RIL population (268 lines) was used to extract DNA for the genotyping of newly developed Kompetitive Allele-Specific PCR (KASP) markers. 


\section{Phenotyping and statistical analysis of bacterial wilt resistance}

The BWR of peanut materials were assessed in two disease nurseries under heavy pressure in Hongan $\left(31.36^{\circ}\right.$ $\mathrm{N}, 114.61^{\circ} \mathrm{E}$, constructed in 1964) and Nanchong, China $\left(30.67^{\circ} \mathrm{N}, 106.06^{\circ} \mathrm{E}\right.$, constructed in 2007). These disease nurseries, in which bacterial wilt disease naturally and evenly occurs across years, were utilized in the BWR evaluation of our previous study (Luo et al. 2019). The RIL population (Xuhua $13 \times$ Zhonghua 6) was planted in a randomized block design with three replications in Hongan during 2015 ( $\mathrm{F}_{6}$ generation $), 2016\left(\mathrm{~F}_{7}\right.$ generation $)$ and $2017\left(\mathrm{~F}_{8}\right.$ generation) as well as in Nanchong during 2015 ( $\mathrm{F}_{6}$ generation) and $2017\left(\mathrm{~F}_{8}\right.$ generation). These five environments were designated as HA2015, HA2016, HA2017, NC2015 and NC2017, respectively. The percentage of plants that were not killed by bacterial wilt disease during the entire period was used to indicate the resistance and referred as survival rate (Luo et al. 2019). Analysis of variance (ANOVA) was performed with the IBM SPSS Statistics version 22 software. The broad-sense heritability for BWR was estimated based on plot mean and entry mean, respectively (Luo et al. 2017).

\section{The improvement of the SSR-based the genetic map}

To improve the previously published SSR-based genetic map (Luo et al. 2018), 456 SSR markers that exhibited polymorphism between Xuhua 13 and Zhonghua 6 were used to genotype the same 187 RILs in $\mathrm{F}_{5}$ generations. These markers were selected according to their positions in the reference genomes of Arachis (Bertioli et al. 2016, 2019) to increase density of the previously published SSR-based genetic map (Luo et al. 2018). PCR amplification and visualization were conducted with the same approach of the previous study (Luo et al. 2018). Similarly, genetic map was re-constructed using the JoinMap 4.0 (Van Ooijen 2006) and visualized with the MapChart 2.3 software (Voorrips 2002). The Kosambi mapping function was used to estimate genetic distances (Kosambi 1943).

\section{QTL mapping using SSR- and SNP-based genetic maps}

Using the improved SSR-based genetic map and the recently reported SNP-based genetic map (Liu et al. 2019), genomewide QTL mapping for BWR was performed using the mean value of survival rates in three replicates in each environment. QTL analysis was conducted by the composite interval mapping (CIM) method using the Windows QTL Cartographer 2.5 software (Wang et al. 2012). The threshold of LOD for declaring the presence of a QTL was determined by 1000 permutation tests. QTLs are designated with an initial letter "q" followed by the trait name (BWR), and the corresponding chromosome, similar to the previously described nomenclature (Luo et al. 2019). The codes " -1 " and " -2 " were added for QTLs detected on the same chromosome. QTLs with PVE higher than $10 \%$ were considered as major otherwise as minor. In addition, a QTL was considered as stable if it was identified across five environments.

\section{Identification of genomic regions for bacterial wilt resistance with the QTL-seq approach}

The QTL-seq approach (Takagi et al. 2013) was adopted to identify the genomic regions for BWR following the pipeline of the previous study (Luo et al. 2019) with a few modifications (as shown in Figure S1). Briefly, 25 RILs with extremely high or low mean survival rates were used to construct the resistant bulk (RB) or susceptible bulk (SB), respectively. Pair-ended reads ( $150 \mathrm{bp}$ ) were generated using the Illumina Hiseq platforms for RB, SB, susceptible parent (SP) and resistant parent (RP). The genome assembly of the cultivated peanut Fuhuasheng (Chen et al. 2019) was downloaded from the NCBI website and used as the reference to develop reference-guided parental assemblies, i.e., the reference-guided assembly for the susceptible parent Xuhua 13 (the SP assembly) and for the resistant parent Zhonghua 6 (the RP assembly). This was the major difference from the previous study (Luo et al. 2019). The high-quality reads of SB and RB were equalized and aligned to the parental assembly to identify SNPs. The $\triangle$ SNP-index for each SNP was calculated by subtracting SNP-index of SB from SNPindex of RB (SNP-index $=\frac{\text { Count of alternate allele }}{\text { Total }}$ ). The sliding window analyses for SNP-index and $\Delta$ SNP-index were conducted with $1 \mathrm{Mb}$ interval and $50 \mathrm{~kb}$ increment to identify genomic regions for BWR.

\section{Marker development and validation}

To validate the identified genomic regions for BWR, KASP markers (Semagn et al. 2014) were developed for the significant SNPs using the genomic sequences of Fuhuasheng (Chen et al. 2019). Each KASP marker was firstly validated with Xuhua 13 and Zhonghua 6 and then used to genotype the 268 lines of the RIL population. The genotyping of KASP markers was conducted at the China Golden Marker (Beijing) Biotech Co., Ltd. In addition, SSR markers within the candidate genomic regions were also used to genotype the 268 RILs. Local genetic maps were constructed with the JoinMap 4.0 software (Van Ooijen 2006) and used to identify QTLs for BWR by both single-marker analysis (SMA) and composite interval mapping (CIM) methods of the Windows QTL Cartographer 2.5 software (Wang et al. 2012). 


\section{Candidate genes analysis}

To identify candidate genes, effective SNPs associated with BWR were identified following the same procedure of our previous study (Luo et al. 2019). The SnpEff v3.0 software (Cingolani et al. 2012) was then used to predict the functions of these effective SNPs based on the published annotation of the genomic sequences of Fuhuasheng (Chen et al. 2019). The putative genes affected by non-synonymous SNPs were selected as candidates for the BWR. The function of candidate genes were predicted using the Blast2GO (Gotz et al. 2008) and eggNOG-mapper tool (Huerta-Cepas et al. 2016). The InterPro online server (Finn et al. 2017) was used to predict the compositions of conserved domains encoded by candidate genes.
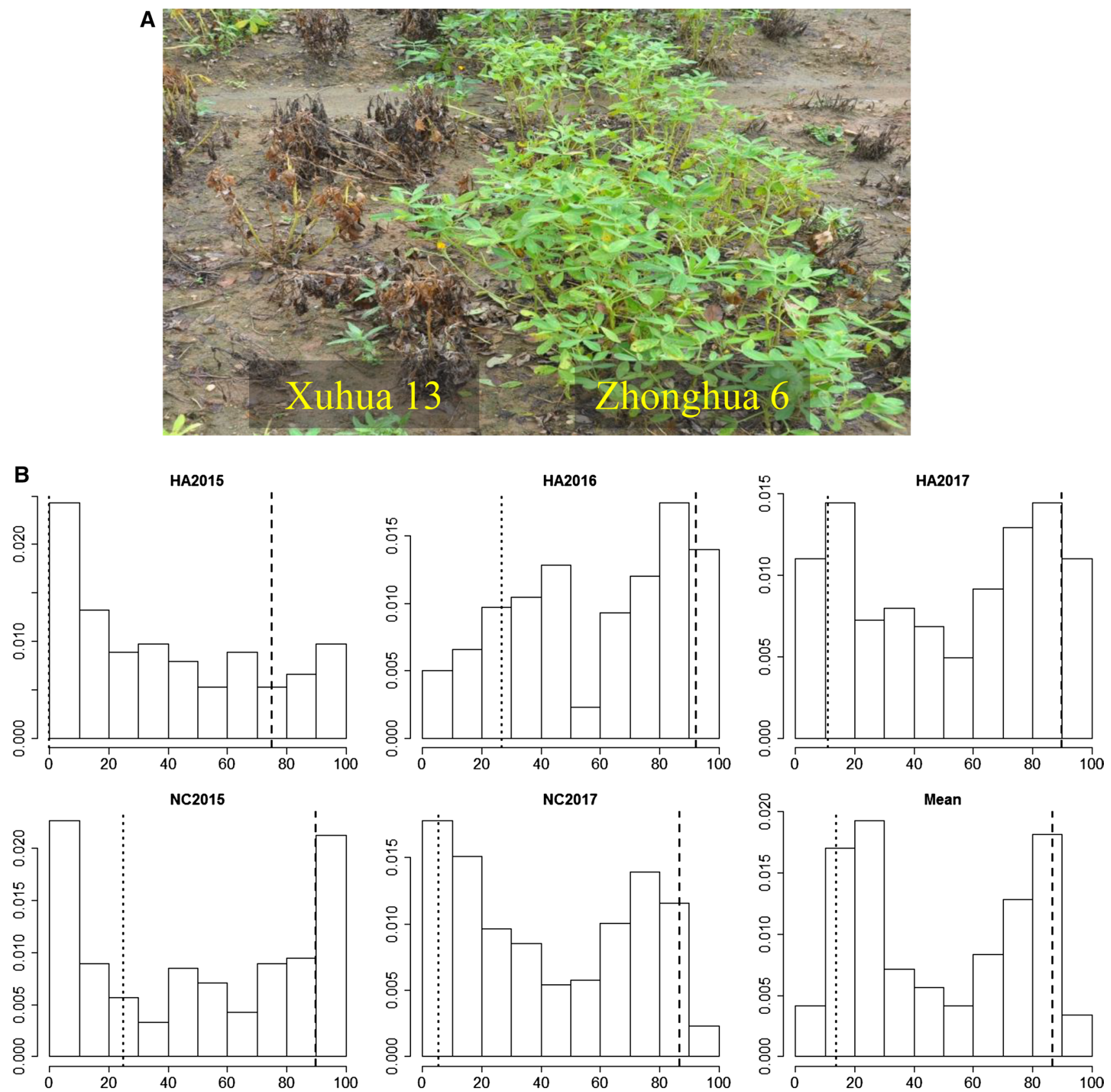

Fig. 1 Phenotypic variations of the RIL population for bacterial wilt resistance across five environments. a The difference of survival rate of susceptible parent Xuhua 13 and resistant parent Zhonghua 6 in the disease nursery, $\mathbf{b}$ frequency distribution for survival rates in RIL population. The $y$-axis represented density, while the $x$-axis represented values of survival rates. The dotted line represented the sur- vival rate of Xuhua 13, and the dashed line represented the survival rate of Zhonghua 6. The HA2015, HA2016, HA2017, NC2015 and NC2017 indicated the phenotyping were conducted in Hongan city (HA) during 2015, 2016, 2017 and Nanchong city (NC) during 2015, 2017 


\section{Results}

\section{Phenotypic variations of BWR in the RIL population}

The resistant parent Zhonghua 6 (RP) showed significantly higher survival rates $(86.75 \%$ on average) than the susceptible parent Xuhua 13 (SP) (13.69\% on average) across five environments (Fig. 1). The survival rates of the 268 RILs showed continuous distributions with two peaks (Fig. 1b), indicating the existing of major QTLs for BWR. Analysis of variance revealed that genotype, environment and genotype $\times$ environment interactions significantly $(P<0.001)$ influenced BWR (Table S1). Broad-sense heritability for BWR was estimated to be $71.07 \%$ based on plot mean and $93.72 \%$ based on entry mean, indicating strong control by genetic factors.

\section{Development of high-density SSR-based genetic map}

A total of 180 SSR markers (Table S2) were successfully added to the previously reported SSR-based genetic map (Luo et al. 2018). Five markers amplified two genetic loci, while the remaining 175 markers amplified single locus (Table S3). The improved SSR-based genetic map consisted of 1002 loci spanning $1838.10 \mathrm{cM}$ with an average inter-marker distance of $1.83 \mathrm{cM}$ (Figure S2, Table S4). The newly genotyped 185 loci were mapped across genome except chromosomes A01, B01, B03 and B09. The improved SSR-based genetic map had 516 loci for the A subgenome and 486 loci for the B subgenome with map lengths of 948.55 and $889.55 \mathrm{cM}$, respectively. The length of linkage groups varied from $32.18 \mathrm{cM}$ (A04) to $134.71 \mathrm{cM}$ (B03), and the number of loci ranged from 7 to 105 markers (Table S5).

\section{Discovery of genome-wide QTLs for BWR using SSR- or SNP-based genetic map}

Genome-wide QTL analysis was performed using the improved SSR-based genetic map and phenotypic data of BWR obtained for the subset (187 RILs) of the mapping population in five environments. Through composite interval mapping (CIM), one major QTL was identified on chromosomes B02 while two minor QTLs were identified on B02 and A07 (Table 1, Figure S3). The major QTL, qBWRB02-1 on the chromosome B02, was stable in expression across five environments with $53.93-78.86 \%$ phenotypic variation explained (PVE) while the two minor QTLs, $q B W R B 02-2$ and $q B W R A 07$, were only expressed in the HA2017 environment (3.78-4.31\% PVE). According to e-PCR locations of the four flanking SSR markers (AGGS1419, GM2196, AHGS2344 and pPGSseq11H1-1) on the genome assembly of Fuhuasheng (Chen et al. 2019), qBWRB02-1 was probably located in a $6.90 \mathrm{Mb}$ interval $(0-6.90 \mathrm{Mb})$ on chromosome B02 (Fig. 2).

Genome-wide QTL analysis was performed using the recently constructed SNP-based genetic map (Liu et al. 2019) as well. This result confirmed the presence of the major and stable QTL $q B W R B 02-1$ on chromosome B02 (37.79-53.85\% PVE), which was estimated to be located in a $7.51 \mathrm{Mb}$ interval $(0.59-8.45 \mathrm{Mb})$ through BLASTn the RAD tags of five flanking SNPs (AhEX369227, AhMXZ237349, AhEXZ392990, AhEXZ131655 and AhEXZ253127) to the genome sequence of Fuhuasheng (Table 2; Fig. 2). Two additional minor QTLs (3.75\% or 5.88\% PVE) were identified on chromosome B01 (Table 2; Figure S4). The minor QTL $q B W R B 01-1$ was identified in the HA2015 environment, while the other minor QTL $q B W R B 01-2$ was identified using the mean values of survival rates across five environments.

Table 1 QTL mapping for bacterial wilt resistance using the improved SSR-based genetic map

\begin{tabular}{lllllllll}
\hline QTL & Env $^{\mathrm{a}}$ & Chr $^{\mathrm{b}}$ & $\begin{array}{l}\text { Genetic } \\
\text { position } \\
(\mathrm{cM})\end{array}$ & LOD value & Marker interval & Physical interval (Mb) & Additive effect $^{\text {PVE }^{\mathrm{c}}(\%)}$ \\
\hline qBWRB02-1 & HA2015 & B02 & 7.21 & 35.81 & AGGS1419-pPGSseq11H1-1 & $0-6.90$ & 25.20 & 60.17 \\
& HA2016 & B02 & 8.21 & 34.26 & AGGS1419-pPGSseq11H1-1 & $0-6.90$ & 20.46 & 53.93 \\
& HA2017 & B02 & 7.21 & 36.84 & AGGS1419-pPGSseq11H1-1 & $0-6.90$ & 23.65 & 54.56 \\
& NC2015 & B02 & 7.21 & 32.28 & AGGS1419-pPGSseq11H1-1 & $0-6.90$ & 29.76 & 58.17 \\
& NC2017 & B02 & 8.21 & 47.91 & AGGS1419-pPGSseq11H1-1 & $0-6.90$ & 24.49 & 69.33 \\
& Mean & B02 & 8.21 & 61.44 & AGGS1419-pPGSseq11H1-1 & $0-6.90$ & 25.38 & 78.86 \\
qBWRB02-2 & HA2017 & B02 & 64.41 & 4.02 & AGGS1635-2-AHGS1241 & $107.50-111.70$ & 6.22 & 3.78 \\
\hline
\end{tabular}

${ }^{a}$ Environment

${ }^{\mathrm{b}}$ Chromosome

${ }^{\mathrm{c}}$ Phenotypic variation explained 


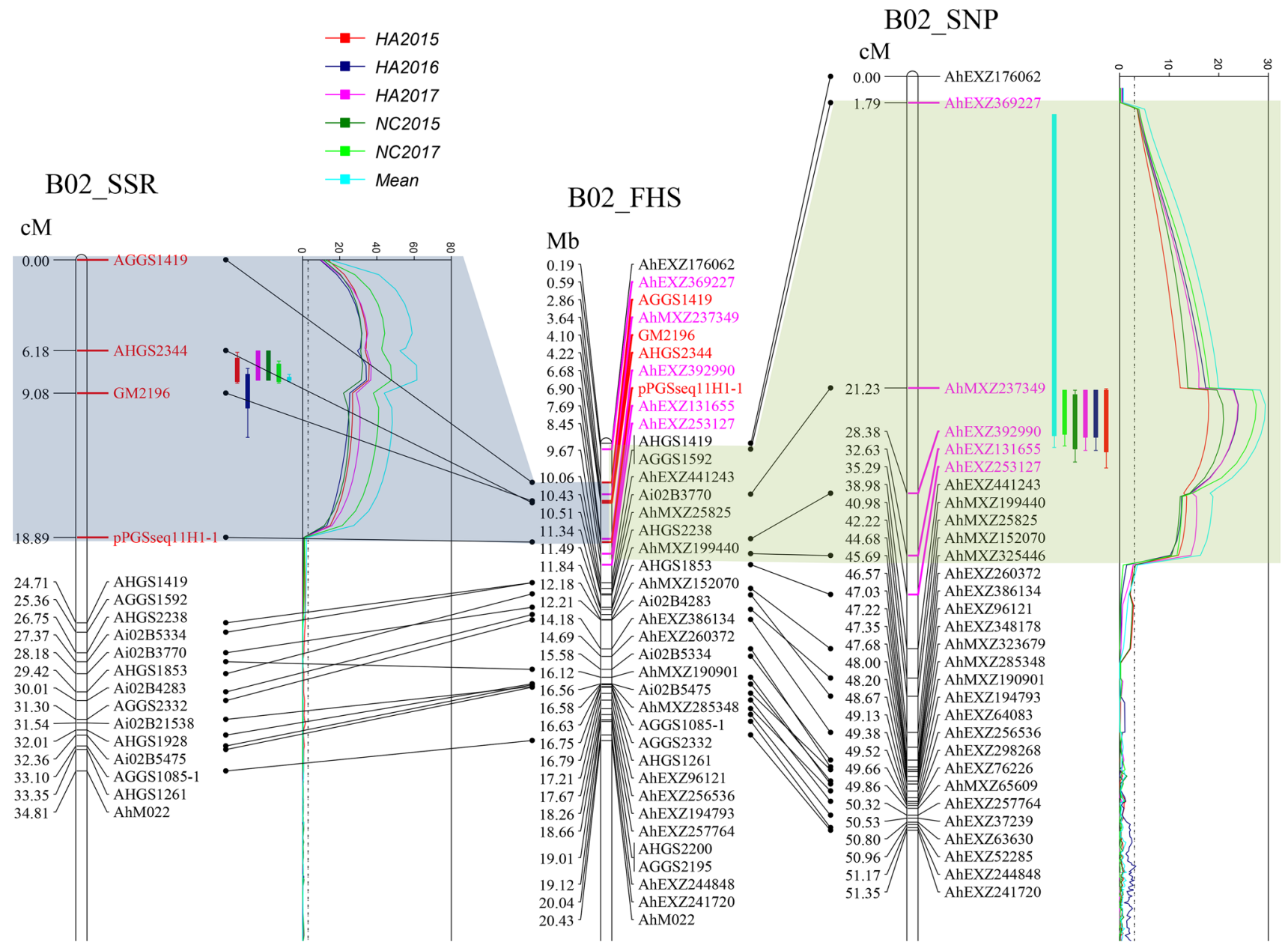

Fig. 2 Co-localization of the major QTL identified by SSR- and SNPbased genetic map for bacterial wilt resistance on chromosome B02. B02_SSR indicates the linkage map B02 constructed based on SSR markers. B02_SNP indicates the reversed linkage map B02 constructed based on SNP loci by Liu et al. (2019). B02_FHS indicates the estimated physical positions of SSR and SNP loci on the genomic sequence of chromosome B02 of Fuhuasheng. The positions of QTL intervals were highlighted in blue and green color, respectively (color figure online)

Table 2 QTL mapping for bacterial wilt resistance using the SNP-based genetic map

\begin{tabular}{lllllllll}
\hline QTL & Env $^{\mathrm{a}}$ & Chr $^{\mathrm{b}}$ & $\begin{array}{l}\text { Genetic } \\
\text { position } \\
(\mathrm{cM})\end{array}$ & LOD value & Marker interval & Physical interval (Mb) & Additive effect PVE $^{\mathrm{c}}(\%)$ \\
\hline$q$ qWRB02-1 & HA2015 & B02 & 91.11 & 17.98 & AhEXZ253127-AhEXZ369227 & $0.59-8.45$ & 19.80 \\
& HA2016 & B02 & 91.11 & 23.94 & AhEXZ253127-AhEXZ369227 & $0.59-8.45$ & 19.44 \\
& HA2017 & B02 & 91.11 & 23.84 & AhEXZ253127-AhEXZ369227 & $0.59-8.45$ & 21.28 & 45.79 \\
& NC2015 & B02 & 91.11 & 20.98 & AhEXZ253127-AhEXZ369227 & $0.59-8.45$ & 26.68 & 47.80 \\
& NC2017 & B02 & 91.11 & 27.57 & AhEXZ253127-AhEXZ369227 & $0.59-8.45$ & 21.36 & 51.88 \\
& Mean & B02 & 91.11 & 29.39 & AhEXZ253127-AhEXZ369227 & $0.59-8.45$ & 21.08 & 53.85 \\
qBWRB01-1 & HA2015 & B01 & 48.51 & 4.81 & AhEXZ281542-AhMXZ205274 & $83.92-84.88$ & 10.30 & 5.88 \\
qBWRB01-2 & Mean & B01 & 45.41 & 4.48 & AhEXZ117519-AhMXZ288911 & $62.04-65.73$ & 7.19 & 3.75 \\
\hline
\end{tabular}

\section{${ }^{\mathrm{a}}$ Environment}

${ }^{\mathrm{b}}$ Chromosome

${ }^{\mathrm{c}}$ Phenotypic variation explained 
Therefore, based on the comparison between intervals identified by SSR- and SNP-based genetic maps, the major and stable QTL $q B W R B 02-1$ was estimated to be located within a $6.31 \mathrm{Mb}$ physical distance $(0.59-6.90 \mathrm{Mb}$, between AhEXZ369227 and AhEXZ392990) on chromosome B02 (Fig. 2).

\section{Identification of genomic regions for BWR by QTL-seq using the tetraploid reference genome}

Based on the mean survival rates of the whole population (268 RILs) across five environments, 25 RILs with survival rates of $5.99-14.37 \%$ and 25 RILs with survival rates of
Fig. 3 Construction of extreme bulks and identification of genomic regions for bacterial wilt resistance by the QTL-seq approach. a Phenotypic variability among the RILs selected for development of extreme bulks for bacterial wilt resistance. Based on mean values of the five environments, 25 RILs with lowest survival rates and 25 RILs with highest survival rates were used to constitute susceptible and resistant bulks, b genomic regions for bacterial wilt resistance identified by QTL-seq using the Xuhua 13 as reference, $\mathbf{c}$ genomic regions for bacterial wilt resistance identified by QTL-seq using the Zhonghua 6 as reference
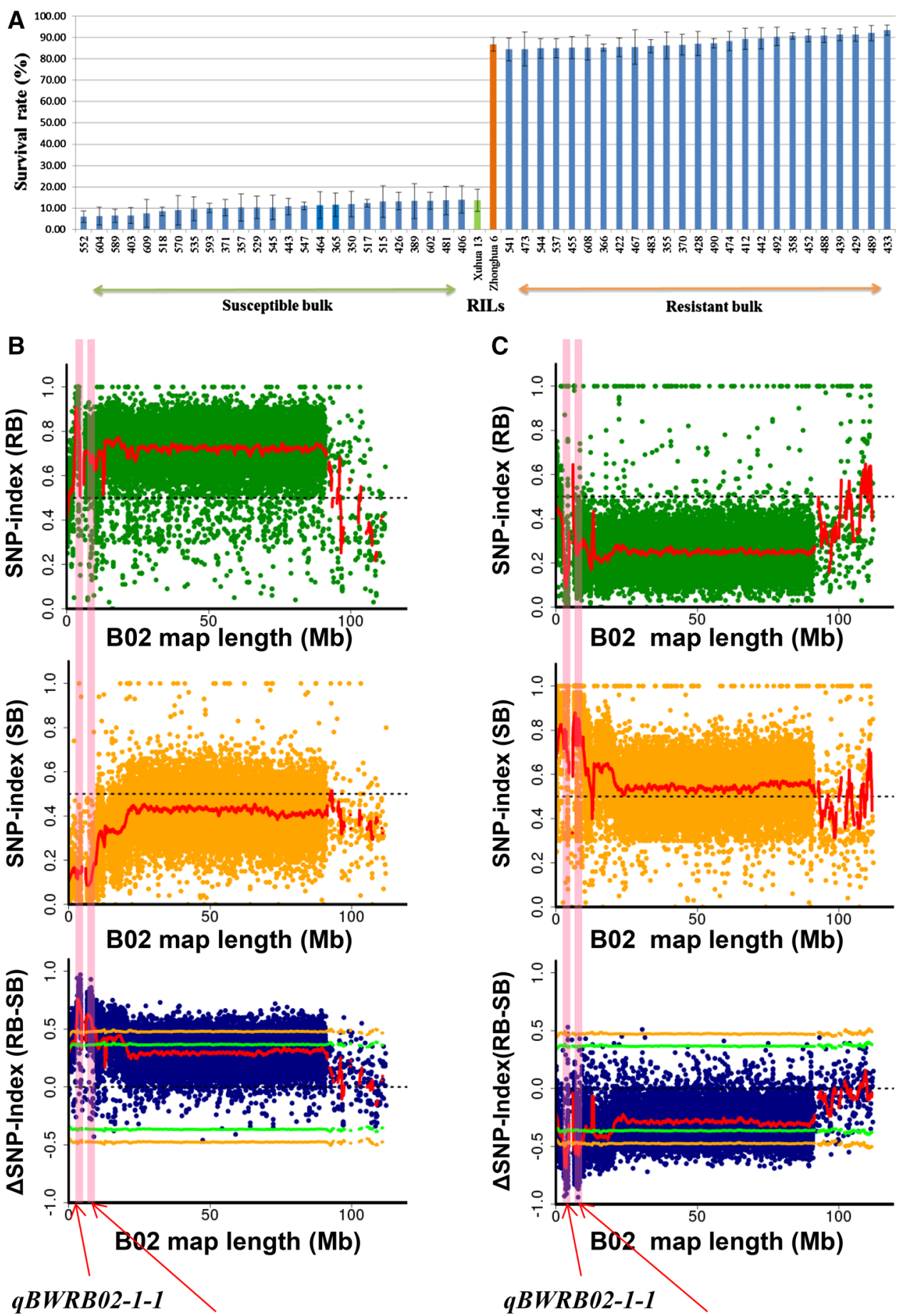

qBWRB 02-1-2

Xuhua 13 as reference parent

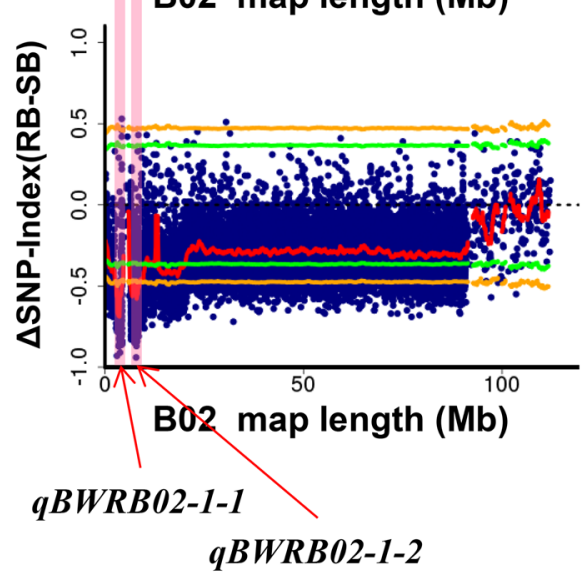

Zhonghua 6 as reference parent 
84.93-95.42\% were used to prepare the susceptible bulk (SB) and resistant bulk (RB), respectively (Fig. 3a). Wholegenome resequencing data, including 936.70 million reads $(140.51 \mathrm{~Gb})$ for susceptible parent (SP, Xuhua 13), 527.10 million reads $(79.06 \mathrm{~Gb})$ for resistant parent $(\mathrm{RP}$, Zhonghua 6), 967.16 million reads $(145.07 \mathrm{~Gb})$ for $\mathrm{RB}$ and 962.28 million reads $(144.34 \mathrm{~Gb})$ for SB (Table 3; Table S6), were generated using Illumina platforms.

Initially, a reference-guided assembly was developed for Xuhua 13 by aligning its reads $(96.75 \%$ genome coverage and 26.79 mean read depth) to the genome assembly of Fuhuasheng (Chen et al. 2019), which was then referred to as the SP assembly (Figure S1). The reads of the RB and SB were mapped to the SP assembly, and achieved $97.20 \%$ mapping coverage and 38.21 mean read depth for the RB while $97.19 \%$ coverage and 36.74 mean read depth for the SB (Table 3, Table S6). A total of 303,290 genome-wide SNPs were identified (Table S7). Based on the sliding window analysis of their SNP-indexes and $\triangle$ SNP-indexes, two major peaks on chromosome B02 were identified for BWR at a statistical confidence of $P<0.01$, spanning $1.47 \mathrm{Mb}$ (2.80-4.27 Mb) and 3.54 Mb (6.11-9.65 Mb) intervals, respectively (Fig. 3b, Figure S5-S7). Their $\Delta$ SNP-indexes were positive, indicating that more alleles were from the non-reference parent Zhonghua 6 in the RB (Table 4).

Similarly, a reference-guided assembly was developed for Zhonghua 6 by aligning its reads $(96.35 \%$ genome coverage and 18.89 mean read depth) to the genome assembly of Fuhuasheng, which was then referred to as the RP assembly (Figure S1). The reads of the RB and SB were also mapped to the RP assembly and achieved 97.20\% coverage and 38.19 mean read depth for the RB while $97.19 \%$ coverage and 37.12 mean read depth for the SB (Table 3; Table S6). A total of 320,522 genome-wide SNPs (Table S7) were identified, among which 281,637 SNPs were also identified in the above analysis using the $\mathrm{SP}$ assembly as reference. Based on the sliding window analysis, two similar genomic regions on chromosome B02 were identified for BWR, i.e., a $1.43 \mathrm{Mb}(2.81-4.24 \mathrm{Mb})$ interval and a $2.21 \mathrm{Mb}(6.54-8.75 \mathrm{Mb})$ interval (Fig. 3c;
Table 3 Summary of survival rates and Illumina sequencing of parental lines and bulks

\begin{tabular}{llllll}
\hline Sample & $\begin{array}{l}\text { Mean survival } \\
\text { rates (\%) }\end{array}$ & $\begin{array}{l}\text { Number of } \\
\text { reads generated }\end{array}$ & Total bases & $\begin{array}{l}\text { Genome cov- } \\
\text { erage (\%) }\end{array}$ & Mean depth $(X)$ \\
\hline Xuhua 13 & 13.69 & $936,700,922$ & $140,505,138,300$ & 96.75 & 26.79 \\
$\mathrm{RB}^{\mathrm{b}}$ & 87.86 & $967,161,084$ & $145,074,162,600$ & 97.20 & 38.21 \\
$\mathrm{SB}^{\mathrm{b}}$ & 10.47 & $962,279,282$ & $144,341,892,300$ & 97.19 & 36.74 \\
Zhonghua 6 $^{\mathrm{a}}$ & 86.75 & $527,095,770$ & $79,064,365,500$ & 96.35 & 18.89 \\
$\mathrm{RB}^{\mathrm{c}}$ & 87.86 & $967,161,084$ & $145,074,162,600$ & 97.20 & 38.19 \\
$\mathrm{SB}^{\mathrm{c}}$ & 10.47 & $962,279,282$ & $144,341,892,300$ & 97.19 & 37.12 \\
\hline
\end{tabular}

${ }^{a}$ The short reads of parental lines were aligned to the published genome sequences of Fuhuasheng

${ }^{\mathrm{b}}$ The short reads of the extreme bulks were aligned to the SP "reference sequence" developed by replacement of SNPs between Xuhua 13 and Fuhuasheng

"The short reads of bulks were aligned to the RP "reference sequence" developed by replacement of SNPs between Zhonghua 6 and Fuhuasheng

\begin{tabular}{lllcccc}
\hline Reference assembly & Genomic region $(\mathrm{Mb})$ & Length $(\mathrm{Mb})$ & $\Delta$ SNP-index $^{\mathrm{d}}$ & U99 $^{\mathrm{e}}$ & L99 $^{\mathrm{f}}$ & Allele source \\
\hline $\mathrm{SP}^{\mathrm{a}}$ & $2.80-4.27$ & 1.47 & 0.76 & 0.49 & -0.49 & Zhonghua 6 \\
& $6.11-9.65$ & 3.54 & 0.63 & 0.48 & -0.48 Zhonghua 6 \\
$\mathrm{RP}^{\mathrm{b}}$ & $2.81-4.24$ & 1.43 & -0.69 & 0.48 & -0.48 Zhonghua 6 \\
& $6.54-8.75$ & 2.21 & -0.58 & 0.47 & -0.48 Zhonghua 6 \\
Combined $^{\mathrm{c}}$ & $2.81-4.24$ & 1.43 & & & & Zhonghua 6 \\
& $6.54-8.75$ & 2.21 & & & & Zhonghua 6 \\
\hline
\end{tabular}

\footnotetext{
${ }^{\mathrm{a}}$ The susceptible parent Xuhua 13

${ }^{\mathrm{b}}$ The resistant parent Zhonghua 6

${ }^{\mathrm{c}}$ The genomic regions determined based on the results of SP and RP

${ }^{\mathrm{d}}$ The highest $\Delta$ SNP-index (SNP-index of resistant bulk - SNP index of susceptible bulk) of widows in the identified genomic region

e $99 \%$ confidence interval upper side

f $99 \%$ confidence interval lower side
} 
Figure S8-S10). The $\Delta$ SNP-indexes of the two genomic regions were negative, indicating that more alleles were from reference parent Zhonghua 6 in the RB (Table 4).

When combined the results of both linkage mapping and QTL-seq, the above genomic regions were overlapped with the major and stable QTL $q B W R B 02-1$. Therefore, two adjacent genomic regions were selected as the candidates for BWR, i.e., 2.81-4.24 $\mathrm{Mb}$ and $6.54-8.75 \mathrm{Mb}$ on B02 for further detailed analysis, and they were refereed as $q B W R B 02-1-1$ and $q B W R B 02-1-2$, respectively (Table 4).

\section{Validation of genomic regions using newly developed KASP markers}

To validate $q B W R B 02-1-1,100$ SNPs with the top $\triangle$ SNPindexes were targeted for KASP marker development. The flanking $200 \mathrm{bp}$ sequences of 27 SNPs were found to be specific when BLASTn to the Fuhuasheng reference genome. Finally, 11 KASP markers were successfully developed for qBWRB02-1-1 (Table S8). Similarly, 100 SNPs with the top $\triangle$ SNP-indexes for $q B W R B 02-1-2$ were targeted for KASP marker development, and 15 KASP markers were successfully developed for $q B W R B 02-1-2$ (Table S8).

Genotyping of 268 RILs was performed with the 26 KASP markers and 4 SSR markers within the genomic region of $q B W R B 02-1-1$ and $q B W R B 02-1-2$. Based on the results of the 30 markers, a genetic map was constructed with map length of $19.60 \mathrm{cM}$ (Table S9). Single-marker analysis (SMA) confirmed the significant associations between BWR and the 30 markers across five environments (Table S9). Among the 13 markers for $q B W R B 02-1-1$, the KASP marker CM014326.1_4160298 was the most significant, with LOD scores of 36.02-54.64. Among the 17 markers for $q B W R B 02-1-2$, the KASP marker CM014326.1_6709822 was the most significant, with LOD scores of 15.19-22.97. CIM analysis indicated that the major and stable effects (49.43-59.90\% PVE) of $q B W R B 02-1-1$ while the minor effects (3.96-6.35\% PVE) of $q B W R B 02-1-2$ across five environments (Table 5).

To evaluate the combined effects of $q B W R B 02-1$ 1 and $q B W R B 02-1-2$, RILs were classified into four groups according to the genotypes of the KASP markers CM014326.1_4160298 and CM014326.1_6709822. The alleles of CM014326.1_4160298 and CM014326.1_6709822 from Zhonghua 6 were designated as "AA" and "BB," respectively, while those from Xuhua 13 were designated as "aa" and "bb." RILs with the AABB and AAbb genotypes showed significantly higher survival rates than those with the aaBB and aabb genotypes in all five environments (Fig. 4, Table S10). The average survival rate of RILs with the AABB genotypes was higher than RILs with the AAbb genotypes but not significantly. Similarly, the average survival rate of RILs with the aaBB genotypes was higher than RILs with the aabb genotype but not significantly. These results indicated that the resistance against bacterial wilt of Zhonghua 6 was mainly controlled by the $q B W R B 02-1-1$.

\section{Putative candidate genes for BWR}

The $1.43 \mathrm{Mb}$ genomic region of $q B W R B 02-1-1$ had 205 effective SNPs. Of the 205 SNPs, 124 SNPs were identified irrespective of which parent was used as reference;

Table 5 Validation of the genomic regions identified by QTL-seq approach on chromosome B02

\begin{tabular}{lllllll}
\hline QTL & Env $^{\mathrm{a}}$ & Marker interval & Most significant marker & LOD value & Additive effect & PVE $^{\mathrm{b}}$ (\%) \\
\hline qBWRB02-1-1 & HA2015 & CM014326.1-4120413-AHGS2344 & CM014326.1-4160298 & 36.65 & 24.98 & 59.37 \\
& HA2016 & CM014326.1-4120413-AHGS2344 & CM014326.1-4160298 & 36.97 & 21.24 & 53.06 \\
& HA2017 & CM014326.1-4120413-AHGS2344 & CM014326.1-4160298 & 38.22 & 22.94 & 52.40 \\
& NC2015 & CM014326.1-4120413-AHGS2344 & CM014326.1-4160298 & 31.60 & 26.30 & 49.43 \\
& NC2017 & CM014326.1-4120413-AHGS2344 & CM014326.1-4160298 & 47.09 & 23.71 & 59.99 \\
& Mean & CM014326.1-4120413-AHGS2344 & CM014326.1-4160298 & 56.85 & 24.02 & 68.86 \\
qBWRB02-1-2 & HA2015 & CM014326.1-6556572-pPGseq11H1-1 & CM014326.1-6709822 & 3.88 & 7.65 & 4.03 \\
& HA2016 & CM014326.1-6556572-pPGseq11H1-1 & CM014326.1-6709822 & 4.22 & 7.19 & 4.43 \\
& HA2017 & CM014326.1-6556572-pPGseq11H1-1 & CM014326.1-6709822 & 6.55 & 10.03 & 6.35 \\
& NC2015 & CM014326.1-6556572-pPGseq11H1-1 & CM014326.1-6709822 & 4.62 & 9.74 & 5.19 \\
& NC2017 & CM014326.1-6556572-pPGseq11H1-1 & CM014326.1-6709822 & 4.42 & 7.31 & 8.54 \\
\hline
\end{tabular}

${ }^{\mathrm{a}}$ Environment

${ }^{\mathrm{b}}$ Phenotypic variation explained 


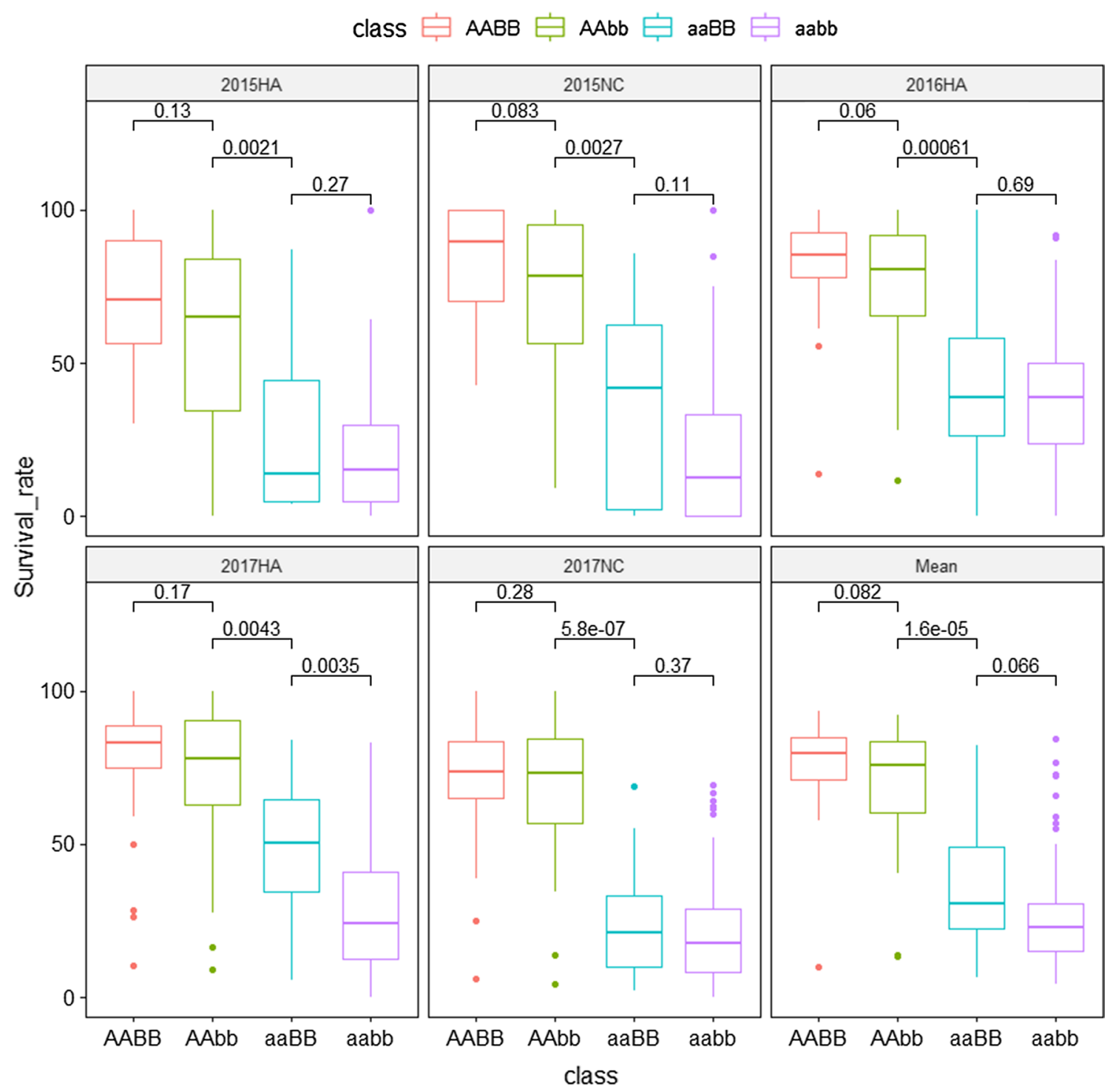

Fig. 4 Boxplots for comparing the difference of survival rates between RILs with different genotypes. Boxplots were generated with the ggpubr package in $\mathrm{R}$ software. The $x$-axis represented the class of genotypes, while the $y$-axis represented values of survival rates

however, 47 SNPs were specifically identified when the SP assembly was used as the reference while 34 SNPs were specifically identified when the RP assembly was used as the reference (Tables S11-12). Function annotation analysis of the 205 SNPs found that 30 SNPs were intergenic while the other 175 SNPs were located in genic regions of 50 putative genes. Among the 175 SNPs, 61 were located in $5 \mathrm{~kb}$ upstream, 3 in $5^{\prime}$ UTR, 23 intronic, 13 synonymous, 49 non-synonymous ( 1 resulted in stop codon) and 26 in $5 \mathrm{~kb}$ downstream. The 49 non-synonymous SNPs affected 16 putative candidate genes for BWR (Table 6). Notably, ten of the 16 candidate genes were predicted to code for disease resistance proteins, including Ahy_B02g057386,
(\%). In each box, centerline shows the median; box limits indicate the 25th and 75th percentiles; whiskers extend 1.5 times the interquartile range from the 25 th and 75 th percentiles. The significant levels of differences between each group were illustrated as $P$ values

Ahy_B02g057483, Ahy_B02g057484, Ahy_B02g057488, Ahy_B02g057489, Ahy_B02g057492, Ahy_B02g057531, Ahy_B02g057533, Ahy_B02g057534 and Ahy_B02g057535 (Figure S11). The candidate gene Ahy_BO2g057524 codes for protein ENHANCED DISEASE RESISTANCE. The candidate genes Ahy_B02g057494 and Ahy_B02g057523 code for pentatricopeptide repeat superfamily protein. The candidate genes Ahy_B02g057498 and Ahy_B02g057528 code for serine threonine-protein phosphatase 7 long form homolog and hydroquinone glucosyltransferase, respectively. However, the candidate gene Ahy_BO2g057521 codes for protein with unknown function. 
Table 6 Non-synonymous SNPs in putative candidate genes in the genomic region for bacterial wilt resistance on chromosome B02

\begin{tabular}{|c|c|c|c|c|c|c|c|c|}
\hline Gene & Position (bp) & SP base & RP base & SB base & $\mathrm{RB}$ base & Amino acid change & Function & $\begin{array}{l}\text { Reference } \\
\text { assembly }^{\mathrm{a}}\end{array}$ \\
\hline Ahy_B02g057386 & 2816632 & $\mathrm{~T}$ & G & $\mathrm{T}$ & $\mathrm{G}$ & Leu1443Phe & Disease resistance protein & Both \\
\hline \multirow[t]{12}{*}{ Ahy_B02g057483 } & 3629422 & $\mathrm{C}$ & $\mathrm{T}$ & $\mathrm{C}$ & $\mathrm{T}$ & Lys251Glu & Disease resistance protein & Both \\
\hline & 3629427 & A & $\mathrm{C}$ & A & $\mathrm{C}$ & Arg249Ile & & $\mathrm{RP}$ \\
\hline & 3629435 & A & $\mathrm{C}$ & A & $\mathrm{C}$ & Arg246Ser & & $\mathrm{RP}$ \\
\hline & 3629449 & $\mathrm{C}$ & $\mathrm{G}$ & $\mathrm{C}$ & $\mathrm{G}$ & Val242Leu & & $\mathrm{RP}$ \\
\hline & 3629461 & A & G & A & $\mathrm{G}$ & Tyr238His & & Both \\
\hline & 3629494 & $\mathrm{G}$ & $\mathrm{C}$ & G & $\mathrm{C}$ & Leu227Val & & $\mathrm{RP}$ \\
\hline & 3629516 & A & $\mathrm{T}$ & A & $\mathrm{T}$ & Asp219Val & & Both \\
\hline & 3629517 & $\mathrm{~T}$ & $\mathrm{C}$ & $\mathrm{T}$ & $\mathrm{C}$ & Asp219Val & & Both \\
\hline & 3629533 & $\mathrm{C}$ & G & $\mathrm{C}$ & G & Glu214Gln & & Both \\
\hline & 3629545 & $\mathrm{C}$ & G & $\mathrm{C}$ & G & Ala210Pro & & Both \\
\hline & 3636318 & $\mathrm{~T}$ & $\mathrm{C}$ & $\mathrm{T}$ & $\mathrm{C}$ & Cys658Tyr & & Both \\
\hline & 3637177 & G & A & G & A & Trp375Arg & & Both \\
\hline \multirow[t]{3}{*}{ Ahy_B02g057484 } & 3652428 & A & $\mathrm{T}$ & A & $\mathrm{T}$ & Lys556Met & Disease resistance protein & SP \\
\hline & 3652429 & $\mathrm{C}$ & $\mathrm{T}$ & $\mathrm{C}$ & $\mathrm{T}$ & Lys556Glu & & SP \\
\hline & 3654929 & $\mathrm{C}$ & $\mathrm{G}$ & $\mathrm{C}$ & $\mathrm{G}$ & Asp119Glu & & SP \\
\hline \multirow[t]{2}{*}{ Ahy_B02g057488 } & 3676848 & $\mathrm{~T}$ & A & $\mathrm{T}$ & A & Thr1020Ser & Disease resistance protein & SP \\
\hline & 3677029 & $\mathrm{C}$ & G & $\mathrm{C}$ & G & Asp959Glu & & Both \\
\hline Ahy_B02g057489 & 3695773 & A & $\mathrm{T}$ & A & $\mathrm{T}$ & Ser519Thr & Disease resistance protein & Both \\
\hline \multirow[t]{4}{*}{ Ahy_B02g057492 } & 3722570 & $\mathrm{C}$ & $\mathrm{G}$ & $\mathrm{C}$ & G & Cys952Ser & Disease resistance protein & $\mathrm{RP}$ \\
\hline & 3723795 & G & $\mathrm{T}$ & G & $\mathrm{T}$ & Leu544Ile & & Both \\
\hline & 3723804 & A & G & A & $\mathrm{G}$ & Ser541Pro & & Both \\
\hline & 3724689 & $\mathrm{~T}$ & G & $\mathrm{T}$ & G & Lys246Gln & & SP \\
\hline \multirow[t]{2}{*}{ Ahy_B02g057494 } & 3729082 & A & $\mathrm{C}$ & A & $\mathrm{C}$ & Asp275Glu & $\begin{array}{l}\text { Pentatricopeptide repeat-containing } \\
\text { protein }\end{array}$ & SP \\
\hline & 3729083 & A & $\mathrm{T}$ & A & $\mathrm{T}$ & Leu276Met & & SP \\
\hline Ahy_B02g057498 & 3755618 & $\mathrm{C}$ & $\mathrm{T}$ & $\mathrm{C}$ & $\mathrm{T}$ & $\operatorname{Trp} 254^{\mathrm{a}}$ & $\begin{array}{l}\text { Serine threonine-protein phos- } \\
\text { phatase } 7 \text { long form homolog }\end{array}$ & SP \\
\hline Ahy_B02g057521 & 4025013 & $\mathrm{~T}$ & $\mathrm{C}$ & $\mathrm{T}$ & $\mathrm{C}$ & Gly65Glu & Unknown & $\mathrm{RP}$ \\
\hline Ahy_B02g057523 & 4078542 & $\mathrm{C}$ & $\mathrm{T}$ & $\mathrm{C}$ & $\mathrm{T}$ & Arg253Gln & $\begin{array}{l}\text { Pentatricopeptide repeat-containing } \\
\text { protein }\end{array}$ & SP \\
\hline Ahy_B02g057524 & 4097658 & $\mathrm{~T}$ & A & $\mathrm{T}$ & A & Asp694Val & $\begin{array}{l}\text { Protein ENHANCED DISEASE } \\
\text { RESISTANCE }\end{array}$ & Both \\
\hline Ahy_B02g057528 & 4124792 & $\mathrm{~T}$ & A & $\mathrm{T}$ & A & Glu35Val & hydroquinone glucosyltransferase & Both \\
\hline Ahy_B02g057531 & 4150673 & $\mathrm{~T}$ & G & $\mathrm{T}$ & G & Phe79Leu & Disease resistance protein & Both \\
\hline Ahy_B02g057533 & 4194809 & $\mathrm{~T}$ & $\mathrm{G}$ & $\mathrm{T}$ & G & Phe72Leu & Disease resistance protein & $\mathrm{RP}$ \\
\hline \multirow[t]{9}{*}{ Ahy_B02g057534 } & 4196305 & $\mathrm{C}$ & $\mathrm{G}$ & $\mathrm{C}$ & G & Thr13Arg & Disease resistance protein & Both \\
\hline & 4196326 & $\mathrm{~T}$ & A & $\mathrm{T}$ & A & Phe20Tyr & & Both \\
\hline & 4196332 & $\mathrm{~T}$ & $\mathrm{~A}$ & $\mathrm{~T}$ & A & Phe22Tyr & & SP \\
\hline & 4196338 & A & $\mathrm{G}$ & A & G & Glu24Gly & & SP \\
\hline & 4196344 & $\mathrm{~T}$ & G & $\mathrm{T}$ & G & Cys26Phe & & SP \\
\hline & 4196346 & $\mathrm{C}$ & G & $\mathrm{C}$ & G & Pro27Ala & & Both \\
\hline & 4196403 & G & $\mathrm{C}$ & G & $\mathrm{C}$ & Arg46Gly & & Both \\
\hline & 4196539 & $\mathrm{~T}$ & G & $\mathrm{T}$ & G & Phe91Cys & & Both \\
\hline & 4196850 & $\mathrm{~T}$ & G & $\mathrm{T}$ & G & Tyr195Asp & & Both \\
\hline \multirow[t]{5}{*}{ Ahy_B02g057535 } & 4236081 & G & $\mathrm{C}$ & G & $\mathrm{C}$ & Asn36Lys & Disease resistance protein & Both \\
\hline & 4236149 & G & A & $\mathrm{G}$ & A & His59Arg & & $\mathrm{RP}$ \\
\hline & 4237744 & A & $\mathrm{C}$ & A & $\mathrm{C}$ & Thr591Pro & & $\mathrm{RP}$ \\
\hline & 4238099 & $\mathrm{C}$ & $\mathrm{T}$ & $\mathrm{C}$ & $\mathrm{T}$ & Thr709Ile & & $\mathrm{RP}$ \\
\hline & 4238107 & A & G & A & G & Asn712Ala & & $\mathrm{RP}$ \\
\hline
\end{tabular}


Table 6 (continued)

\begin{tabular}{|c|c|c|c|c|c|c|c|c|}
\hline Gene & Position (bp) & SP base & RP base & SB base & RB base & Amino acid change & Function & $\begin{array}{l}\text { Reference } \\
\text { assembly }^{\mathrm{a}}\end{array}$ \\
\hline & 4238108 & A & $\mathrm{C}$ & A & $\mathrm{C}$ & Asn712Ala & & $\mathrm{RP}$ \\
\hline & 4238109 & $\mathrm{~T}$ & G & $\mathrm{T}$ & G & Asn712Ala & & $\mathrm{RP}$ \\
\hline & 4238112 & $\mathrm{C}$ & G & $\mathrm{C}$ & G & Asn713Lys & & $\mathrm{RP}$ \\
\hline Ahy_B02g057721 & 6817695 & $\mathrm{~T}$ & $\mathrm{C}$ & $\mathrm{T}$ & $\mathrm{C}$ & Ser253Phe & Unknown & Both \\
\hline Ahy_B02g057750 & 7160780 & $\mathrm{C}$ & $\mathrm{T}$ & $\mathrm{C}$ & $\mathrm{T}$ & $\operatorname{Trp} 97^{\mathrm{a}}$ & $\begin{array}{l}\text { Serine threonine-protein phos- } \\
\text { phatase } 7 \text { long form homolog }\end{array}$ & Both \\
\hline Ahy_B02g057762 & 7362813 & G & $\mathrm{T}$ & G & $\mathrm{T}$ & Asp251Ala & transposon protein & Both \\
\hline Ahy_B02g057766 & 7444948 & $\mathrm{C}$ & $\mathrm{T}$ & $\mathrm{C}$ & $\mathrm{T}$ & Asn325Asp & $\begin{array}{l}\text { glucose-6-phosphate 1-dehydroge- } \\
\text { nase }\end{array}$ & Both \\
\hline \multirow[t]{2}{*}{ Ahy_B02g057785 } & 7664044 & $\mathrm{C}$ & $\mathrm{G}$ & $\mathrm{C}$ & $\mathrm{G}$ & Arg191Thr & $\begin{array}{l}\text { Mitochondrial glycoprotein family } \\
\text { protein }\end{array}$ & $\mathrm{RP}$ \\
\hline & 7664059 & G & A & $\mathrm{G}$ & A & Tyr196Cys & & $\mathrm{RP}$ \\
\hline Ahy_B02g057787 & 7681335 & G & A & G & A & Arg338Gly & $\begin{array}{l}\text { Mitochondrial glycoprotein family } \\
\text { protein }\end{array}$ & $\mathrm{RP}$ \\
\hline Ahy_B02g057789 & 7717654 & $\mathrm{~T}$ & $\mathrm{C}$ & $\mathrm{T}$ & $\mathrm{C}$ & Gly308Asp & $\begin{array}{l}\text { Serine threonine-protein phos- } \\
\text { phatase } 7 \text { long form homolog }\end{array}$ & Both \\
\hline Ahy_B02g057800 & 7912404 & G & $\mathrm{T}$ & $\mathrm{G}$ & $\mathrm{T}$ & Val241Gly & $\begin{array}{l}\text { replication protein A } 70 \mathrm{kDa} \text { DNA- } \\
\text { binding subunit E }\end{array}$ & Both \\
\hline Ahy_B02g057801 & 7919763 & $\mathrm{~T}$ & G & $\mathrm{T}$ & G & Asn28Lys & $\begin{array}{l}\text { pre-mRNA-processing factor 39-like } \\
\text { isoform X1 }\end{array}$ & Both \\
\hline Ahy_B02g057808 & 8042482 & $\mathrm{C}$ & $\mathrm{T}$ & $\mathrm{C}$ & $\mathrm{T}$ & Ser457Gly & $\begin{array}{l}\text { Serine threonine-protein phos- } \\
\text { phatase } 7 \text { long form homolog }\end{array}$ & Both \\
\hline Ahy_B02g057809 & 8059557 & $\mathrm{C}$ & $\mathrm{T}$ & $\mathrm{C}$ & $\mathrm{T}$ & Cys248Arg & $\begin{array}{l}\text { Serine threonine-protein phos- } \\
\text { phatase } 7 \text { long form homolog }\end{array}$ & Both \\
\hline \multirow[t]{2}{*}{ Ahy_B02g057838 } & 8253903 & $\mathrm{C}$ & $\mathrm{T}$ & $\mathrm{C}$ & $\mathrm{T}$ & Lys217Glu & $\begin{array}{l}\text { ARID BRIGHT DNA-binding } \\
\text { domain-containing protein }\end{array}$ & Both \\
\hline & 8254004 & $\mathrm{C}$ & $\mathrm{T}$ & $\mathrm{C}$ & $\mathrm{T}$ & Asn183Ser & & Both \\
\hline Ahy_B02g057842 & 8318756 & G & $\mathrm{C}$ & G & $\mathrm{C}$ & Ala692Gly & $\begin{array}{l}\text { E3 ubiquitin-protein ligase } \\
\text { RNF144A-like }\end{array}$ & SP \\
\hline Ahy_B02g057850 & 8407952 & $\mathrm{C}$ & $\mathrm{T}$ & $\mathrm{C}$ & $\mathrm{T}$ & Val123Ala & $\begin{array}{l}\text { E3 ubiquitin-protein ligase } \\
\text { RNF144A-like }\end{array}$ & Both \\
\hline Ahy_B02g057860 & 8554265 & G & $\mathrm{T}$ & G & $\mathrm{T}$ & Tyr329Asp & Unknown & Both \\
\hline Ahy_B02g057386 & 2816632 & $\mathrm{~T}$ & G & $\mathrm{T}$ & G & Leu1443Phe & Disease resistance protein & Both \\
\hline
\end{tabular}

${ }^{a}$ Both indicated that the SNP was identified irrespective of parent used as reference. SP indicated that the SNP was identified specifically with the susceptible parent Xuhua 13 as reference. RP indicated that the SNP was identified specifically with the resistant parent Zhonghua 6 as reference

The 2.21 Mb genomic region of $q B W R B 02-1-2$ had 464 effective SNPs. Of the 464 SNPs, 433 SNPs were identified irrespective of parent used as reference; however, 12 SNPs were specifically identified when SP assembly was used as reference while 19 SNPs were specifically identified when RP assembly was used as reference (Table S1112). Function annotation analysis of the 464 SNPs found that 160 SNPs were intergenic while the other 304 SNPs located in genic region of 112 putative genes. Among the 304 SNPs, 136 were located in $5 \mathrm{~kb}$ upstream, 6 in 5' UTR, 33 intronic, 7 synonymous, 17 non-synonymous ( 1 resulted in stop codon), 3 in $3^{\prime}$ UTR and 102 in $5 \mathrm{~kb}$ downstream. Notably, the 17 non-synonymous SNPs affected 15 putative candidate genes for BWR (Table 6). Two of the 15 candidate genes, Ahy_B02g057721 and Ahy_B02g057860, might code for protein with unknown function. The candidate genes Ahy_B02g057842 and Ahy_B02g057850 code for E3 ubiquitin-protein ligase RNF144A-like proteins. The candidate genes $A h y_{-}$ B02g057785 and Ahy_B02g057787 code for mitochondrial glycoprotein family protein. The candidate genes Ahy_B02g057766, Ahy_B02g057838, Ahy_B02g057762, Ahy_B02g057800 and Ahy_B02g057801 code for glucose6-phosphate 1-dehydrogenase, ARID BRIGHT DNAbinding domain-containing protein, transposon protein, replication protein $\mathrm{A} 70 \mathrm{kDa}$ DNA-binding subunit $\mathrm{E}$ and 
pre-mRNA-processing factor 39-like isoform X1, respectively (Table 6).

\section{Discussion}

Three high-quality reference genomes for both the subspecies of cultivated tetraploids were published in 2019 (Bertioli et al. 2019; Chen et al. 2019; Zhuang et al. 2019). Before this year, we had previously conducted QTL-seq analysis using diploid reference genomes (Bertioli et al. 2016); however, the availability of the tetraploid genome facilitated performing such analysis making it more precise and accurate. Prior to this study, although the genetic and genomic QTL analyses of BWR were performed for two resistant sources namely Yueyou 92 (Zhao et al. 2016) and Yuanza 9102 (Luo et al. 2019), the genetic basis of the majority resistant germplasm could not be dissected. Through the present study, we have successfully performed combination of genetic mapping and QTL-seq analysis using tetraploid reference genome and identified genomic regions, candidate genes and efficient markers for BWR in a promising resistant source Zhonghua 6.

\section{Construction of high-density genetic map in cultivated peanut}

Constructing high-density genetic maps and performing genomic-wide QTL discovery has been the routine approach using bi-parental genetic populations in crop plants, including peanut. Among different marker systems, SSRs were widely used to construct genetic linkage maps and to map QTLs for disease resistance, drought tolerance, quality- and yield-related traits in cultivated peanut since 2009 (Varshney et al. 2009; Vishwakarma et al. 2017). In our previous study (Luo et al. 2018), a SSR-based genetic map of the RIL population derived from Xuhua $13 \times$ Zhonghua 6 was constructed with 817 loci, which has been improved to 1002 loci in the present study. A total of 185 loci were selectively added to 16 of the 20 chromosomes according to the positions of SSRs in the reference genomes of Arachis (Bertioli et al. 2016, 2019). Compared to the reported SSRbased genetic maps (Huang et al. 2016; Yu et al. 2019), the loci number (1200) and density (1.83 cM/locus) of the present genetic map reached a fairly high level for performing precise QTL discovery. However, in recent times, the SNP markers have emerged as choice of markers due to their amenability for high throughput data generation and being cost-effective and time-saving (Pandey et al. 2016). The SNP-based genetic maps were constructed through multiple sequencing-based genotyping methods such as double-digest restriction-site-associated DNA sequencing (ddRADseq) (Zhou et al. 2014), genotyping-by-sequencing
(GBS) (Dodia et al. 2019), specific length amplified fragment sequencing (SLAF-seq) (Hu et al. 2018; Li et al. 2019; Wang et al. 2018b) and whole-genome resequencing (Agarwal et al. 2018). These genetic maps were medium to high density as compared to sparsely dense SSR-based genetic maps. For example, a SNP-based linkage map for the same RIL population (Xuhua $13 \times$ Zhonghua 6 ) was constructed with 2,595 SNP loci through ddRADseq (Liu et al. 2019). These SNP markers were classified into 20 linkage groups according to their RAD tags hits in the reference genomes of A. duranensis and A. ipaensis (Bertioli et al. 2016). Therefore, the published reference genomes of Arachis facilitated the construction of genetic maps in cultivated peanut, and the high-density SSR- and SNP-based genetic maps laid a foundation for the characterization of the genetic components controlling BWR.

\section{Genetic map-based QTL mapping and sequencing-based QTL-seq uncovers candidate genomic regions controlling BWR in a new resistant variety Zhonghua 6}

Genetic factors play major roles in the determination of resistance to bacterial wilt in peanut, which has shown high $(81.72 \%)$ broad-sense heritability in the RIL population of Yuanza 9102 (resistant) $\times$ Xuzhou 68-4 (susceptible) (Wang et al. 2018a). The present study also observed high broadsense heritability for BWR, i.e., 71.07\% based on plot mean and $93.72 \%$ based on entry mean, in another RIL population of Xuhua 13 (susceptible) $\times$ Zhonghua 6 (resistant). In previous studies, the BWR in peanut variety Yueyou 92 was found to be controlled by one major and stable QTL $q B W$ 1 plus one unstable QTL $q B W-2$ (Zhao et al. 2016), and the BWR in Yuanza 9102 was also controlled by one major and stable QTL $q B W B 02.1$ plus three unstable minor QTLs (Wang et al. 2018a). In the present study, genome-wide QTL analysis identified one major and two minor QTLs (Table 1, Figure S3) using the improved SSR-based genetic map. The major QTL $q B W R B 02-1$ was stable in expression across five environments (53.93-78.86\% PVE), while the two minor QTLs were only expressed in single environments (3.78-4.31\% PVE). The major QTL $q B W R B 02-1$ was also identified using the SNP-based genetic map, while two additional minor QTLs (3.75-5.88\% PVE) were identified in single environments (Table 2; Fig. 2; Figure S4). Therefore, major and stable QTLs for BWR exist in peanut, which will be valuable in the breeding of elite varieties with enhance resistance and agronomic traits.

Using the reference genome of the diploid ancestors of cultivated peanut (Bertioli et al. 2016), the QTL-seq approach has proved to be very successful in identifying candidate regions for major and stable QTL $q B W B 02.1$ in Yuanza 9102 (Luo et al. 2019). Recently, the genome 
sequences of the three cultivated peanut genotypes, including Fuhuasheng (Chen et al. 2019), Shitouqi (Zhuang et al. 2019) and Tifrunner (Bertioli et al. 2019), were published and made available to the peanut research community. Based on the pedigree of parental genotypes (Zhonghua 6 and Xuhua 13), the closest tetraploid genome sequence of Fuhuasheng was used as reference in QTL-seq pipeline (Figure S1) to identify the physical interval for the major QTL $q B W R B 02-1$. In the previous report (Luo et al. 2019), there was significant difference of SNPs $(164,522$ vs. 243,380 ) identified using different parental assembly as reference. However, the number and SNPs identified using different parental assembly as reference were quite similar $(303,290$ vs. 320,522$)$, indicating that the improvement of reference genome could make the QTLseq more precise and accurate. Two adjacent genomic regions (2.81-4.24 $\mathrm{Mb}$ and $6.54-8.75 \mathrm{Mb}$ ) on chromosome B02 were identified within the confidential interval of $q B W R B 02-1$, thus designated as $q B W R B 02-1-1$ and $q B W R B 02-1-2$, respectively (Table 4; Fig. 3). Through validation with KASP markers, $q B W R B 02-1-1$ had major effects (49.43-68.86\% PVE) while $q B W R B 02-1-2$ had minor effects (3.96-6.48\% PVE); however, both of them were stable expressed across five environments (Table 5). Different from previous report (Luo et al. 2019), the QTLseq approach could identify candidate regions not only for major QTL but also for minor QTL as long as it is stably expressed across environments.

\section{qBWRB02-1-1 would be a novel and valuable QTL in breeding elite varieties with enhanced BWR}

The major QTL $q B W-1$ identified from Yueyou 92 might derive from Chinese landrace Xiekangqing (Zhao et al. 2016) and locate on chromosome B04 (Luo et al. 2019). Although the major QTL $q B W R B 02.1$ identified from Yuanza 9102 was located on chromosome $\mathrm{B} 02$, the resistant allele might derive from $A$. diogoi through interspecific hybridization according to pedigree analysis and it was absent in Zhonghua 6 and Yueyou 92 according to diagnostic marker detection (Luo et al. 2019). Based on the pedigree tracking, the source of $q B W R B 02-1-1$ identified on chromosome B02 from Zhonghua 6 in the present study was from a Chinese landrace Taishan Zhenzhu. The PVEs (49.43-68.86\%) of $q B W R B 02$ 1-1 were much higher than the previously reported $q B W-1$ in Yueyou 92 (11.9-21.6\% PVE) and $q B W R B 02.1$ in Yuanza 9102 (14.4-29.32\% PVE). Therefore, we assumed that $q B W R B 02-1-1$ should be a novel and valuable QTL, and it is possible to further improve resistance level to bacterial wilt disease by pyramiding diverse resistant QTLs/genes from both wild and cultivated peanut.

\section{Candidate genes identified for qBWRB02-1-1}

Through e-PCR of SSR markers and BLASTn of RAD tags, the physical interval of $q B W R B 02-1-1$ could be estimated but limited information was provided for the identification of candidate genes. Through QTL-seq, high-density SNPs were identified by resequencing of the parental genotypes and extreme pools. Function annotation analysis of nonsynonymous SNPs variations identified 16 putative candidate genes for $q B W R B 02-1-1$ (Table 6). Ten candidate genes, including Ahy_B02g057386, Ahy_BO2g057483, Ahy_B02g057484, Ahy_B02g057488, Ahy_B02g057489, Ahy_B02g057492, Ahy_B02g057531, Ahy_B02g057533, Ahy_BO2g057534 and Ahy_BO2g057535, were predicted to code for disease resistance proteins which play keys role in the plant immune system (Jones and Dangl 2006; Spoel and Dong 2012). These proteins shared typical Rx N-terminal (Rx_N) domain (Hao et al. 2013), nucleotide binding site (NBS) domain (van Ooijen et al. 2008), leucine-rich repeat (LRR) domain (Sanseverino et al. 2010) of R-genes (Figure S11). Notably, maximum number of non-synonymous SNPs (12) was identified in the $A h y \_B 02 g 057483$ gene, flowed by Ahy_B02g057534 (9) and Ahy_B02g057535 (8). The gene Ahy_B02g057524 codes homolog of EDR4 which plays a negative role in disease resistance (Wu et al. 2015). The candidate genes Ahy_B02g057494 and Ahy_B02g057523 code for pentatricopeptide repeat superfamily proteins, whose knockdown mutants displayed more severe disease symptoms when challenged by pathogenic bacteria (Park et al. 2014). The gene Ahy_B02g057498 codes homolog of serine threonine-protein phosphatase 7 long form protein which might take part in hypersensitive response (Zhou et al. 1995). The identified non-synonymous SNPs in the Ahy $B 02 g 057498$ gene resulted in early termination of protein translation. The gene Ahy_BO2g057528 codes homolog of hydroquinone glucosyltransferase whose suppressed expression might improve resistance to bacteria (Park et al. 2011). Therefore, the above genes should be targeted as candidates for fine mapping and functional validation.

In conclusion, the present study identified two novel and adjacent QTLs on chromosome B02 controlling resistance to bacterial wilt disease in peanut-resistant variety Zhonghua 6. Promising candidate genes were identified in these intervals but cannot be overemphasized until validated through fine mapping and/or complementation tests. These genomic regions and validated markers intervals would be of great value for marker-assisted selection to improve BWR in future peanut breeding.

Acknowledgements This study was supported by the National Natural Science Foundations of China (Grant Nos. 31601340, 31571713, $31761143005,31461143022)$, the Youth Chenguang Project of Science and Technology of Wuhan City (Grant No. 2017050304010285), the Natural Science Foundation of Hubei Province (Grant No. 
2017CFB540) and the Central Public-interest Scientific Institution Basal Research Fund (Grant No. 1610172019008). This work has been undertaken as part of the CGIAR Research Program on Grain Legumes and Dryland Cereals (GLDC). ICRISAT is a member of CGIAR Consortium. The funders had no role in study design, data collection and analysis, decision to publish, or preparation of the manuscript.

Author contribution statement HL, MKP, HKS, RKV, YL, BL and HJ conceived, designed and supervised the experiments. XR, YL, BL and HJ developed the RIL population. HL, HZ, SX, XR, JG, LH, XZ, YC, WC and NL conducted field trials and phenotyping. HL, HZ, SX, JG and WC performed DNA extraction and genotyping. HL, MKP, YZ, HKS, BL, RKV and HJ performed the QTL-seq analysis and interpreted the results. HL prepared the first draft, and HL, MKP, HKS, YL, BL, RKV and $\mathrm{HJ}$ contributed to the final editing of manuscript. All authors read and approved the final manuscript.

\section{Compliance with ethical standards}

Conflict of interest The authors declare that there is no conflict of interest.

Open Access This article is licensed under a Creative Commons Attribution 4.0 International License, which permits use, sharing, adaptation, distribution and reproduction in any medium or format, as long as you give appropriate credit to the original author(s) and the source, provide a link to the Creative Commons licence, and indicate if changes were made. The images or other third party material in this article are included in the article's Creative Commons licence, unless indicated otherwise in a credit line to the material. If material is not included in the article's Creative Commons licence and your intended use is not permitted by statutory regulation or exceeds the permitted use, you will need to obtain permission directly from the copyright holder. To view a copy of this licence, visit http://creativecommons.org/licenses/by/4.0/.

\section{References}

Agarwal G, Clevenger J, Pandey MK, Wang H, Shasidhar Y, Chu $Y$ et al (2018) High-density genetic map using whole-genome resequencing for fine mapping and candidate gene discovery for disease resistance in peanut. Plant Biotechnol J 16:1954-1967

Bertioli DJ, Cannon SB, Froenicke L, Huang G, Farmer AD, Cannon EK et al (2016) The genome sequences of Arachis duranensis and Arachis ipaensis, the diploid ancestors of cultivated peanut. Nat Genet 48:438-446

Bertioli DJ, Jenkins J, Clevenger J, Dudchenko O, Gao D, Seijo G et al (2019) The genome sequence of segmental allotetraploid peanut Arachis hypogaea. Nat Genet 51:877-884

Chen X, Lu Q, Liu H, Zhang J, Hong Y, Lan H et al (2019) Sequencing of cultivated peanut, Arachis hypogaea, yields insights into genome evolution and oil improvement. Mol Plant 12:920-934

Cingolani P, Platts A, le Wang L, Coon M, Nguyen T, Wang L et al (2012) A program for annotating and predicting the effects of single nucleotide polymorphisms, SnpEff: SNPs in the genome of Drosophila melanogaster strain $w^{1118}$; iso-2; iso-3. Fly 6:80-92

Deslandes L, Genin S (2014) Opening the Ralstonia solanacearum type III effector tool box: insights into host cell subversion mechanisms. Curr Opin Plant Biol 20:110-117

Dodia SM, Joshi B, Gangurde SS, Thirumalaisamy PP, Mishra GP, Narandrakumar D et al (2019) Genotyping-by-sequencing based genetic mapping reveals large number of epistatic interactions for stem rot resistance in groundnut. Theor Appl Genet 132:1001-1016

FAOSTAT (2019) FAOSTAT. FAO. https://www.fao.org/faostat/ en/\#data/QC. Accessed 07 Oct 2019

Finn RD, Attwood TK, Babbitt PC, Bateman A, Bork P, Bridge AJ et al (2017) InterPro in 2017-beyond protein family and domain annotations. Nucleic Acids Res 45:D190-D199

Gotz S, Garcia-Gomez JM, Terol J, Williams TD, Nagaraj SH, Nueda MJ et al (2008) High-throughput functional annotation and data mining with the Blast2GO suite. Nucleic Acids Res 36:3420-3435

Hao W, Collier SM, Moffett P, Chai JJ (2013) Structural basis for the interaction between the potato virus $\mathrm{X}$ resistance protein $(\mathrm{Rx})$ and its cofactor Ran GTPase-activating protein 2 (RanGAP2). J Biol Chem 288:35868-35876

Hu XH, Zhang SZ, Miao HR, Cui FG, Shen Y, Yang WQ et al (2018) High-density genetic map construction and identification of QTLs controlling oleic and linoleic acid in peanut using SLAF-seq and SSRs. Sci Rep 8:5479

Huang L, Ren X, Wu B, Li X, Chen W, Zhou X et al (2016) Development and deployment of a high-density linkage map identified quantitative trait loci for plant height in peanut (Arachis hypogaea L.). Sci Rep 6:39478

Huerta-Cepas J, Szklarczyk D, Forslund K, Cook H, Heller D, Walter MC et al (2016) eggNOG 4.5: a hierarchical orthology framework with improved functional annotations for eukaryotic, prokaryotic and viral sequences. Nucleic Acids Res 44:D286-293

Janila P, Variath MT, Pandey MK, Desmae H, Motagi BN, Okori P et al (2016) Genomic tools in groundnut breeding program: status and perspectives. Front Plant Sci 7:289

Jiang G, Wei Z, Xu J, Chen H, Zhang Y, She X et al (2017) Bacterial wilt in China: history, current status, and future perspectives. Front Plant Sci 8:1549

Jones JD, Dangl JL (2006) The plant immune system. Nature 444:323-329

Kosambi DD (1943) The estimation of map distances from recombination values. Ann Hum Genet 12:172-175

Li L, Yang X, Cui S, Meng X, Mu G, Hou M et al (2019) Construction of high-density genetic map and mapping quantitative trait loci for growth habit-related traits of peanut (Arachis hypogaea L.). Front Plant Sci 10:745

Liao B (2017) Germplasm characterization and trait discovery in peanut. In: Varshney RK, Pandey MK, Puppala N (eds) The peanut genome. Springer, Cham, pp 53-68. https://doi.org/10.1007/9783-319-63935-2 5

Liu N, Guo J, Zhou X, Wu B, Huang L, Luo H et al (2019) Highresolution mapping of a major and consensus quantitative trait locus for oil content to $\mathrm{a} \sim 0.8-\mathrm{Mb}$ region on chromosome A08 in peanut (Arachis hypogaea L.). Theor Appl Genet. https://doi. org/10.1007/s00122-019-03438-6

Luo H, Xu Z, Li Z, Li X, Lv J, Ren X et al (2017) Development of SSR markers and identification of major quantitative trait loci controlling shelling percentage in cultivated peanut (Arachis hypogaea L.). Theor Appl Genet 130:1635-1648

Luo H, Guo J, Ren X, Chen W, Huang L, Zhou X et al (2018) Chromosomes A07 and A05 associated with stable and major QTLs for pod weight and size in cultivated peanut (Arachis hypogaea L.). Theor Appl Genet 131:267-282

Luo H, Pandey MK, Khan AW, Wu B, Guo J, Ren X et al (2019) Nextgeneration sequencing identified genomic region and diagnostic markers for resistance to bacterial wilt on chromosome B02 in peanut (Arachis hypogaea L.). Plant Biotechnol J. https://doi. org/10.1111/pbi.13153

Mallikarjuna N, Varshney RK (eds) (2014) Genetics, genomics and breeding of peanuts. CRC Press, New York

Pandey MK, Roorkiwal M, Singh VK, Ramalingam A, Kudapa H, Thudi $\mathrm{M}$ et al (2016) Emerging genomic tools for legume 
breeding: current status and future prospects. Front Plant Sci $7: 455$

Park HJ, Kwon CS, Woo JY, Lee GJ, Kim YJ, Paek KH (2011) Suppression of UDP-glycosyltransferase-coding Arabidopsis thaliana UGT74E2 gene expression leads to increased resistance to Psuedomonas syringae pv. tomato DC3000 infection. Plant Pathol J 27:170-182

Park YJ, Lee HJ, Kwak KJ, Lee K, Hong SW, Kang H (2014) MicroRNA400-guided cleavage of pentatricopeptide repeat protein mRNAs renders Arabidopsis thaliana more susceptible to pathogenic bacteria and fungi. Plant Cell Physiol 55:1660-1668

Salanoubat M, Genin S, Artiguenave F, Gouzy J, Mangenot S, Arlat $\mathrm{M}$ et al (2002) Genome sequence of the plant pathogen Ralstonia solanacearum. Nature 415:497-502

Sanseverino W, Roma G, De Simone M, Faino L, Melito S, Stupka E et al (2010) PRGdb: a bioinformatics platform for plant resistance gene analysis. Nucleic Acids Res 38:D814-821

Semagn K, Babu R, Hearne S, Olsen M (2014) Single nucleotide polymorphism genotyping using Kompetitive Allele Specific PCR (KASP): overview of the technology and its application in crop improvement. Mol Breed 33:1-14

Spoel SH, Dong XN (2012) How do plants achieve immunity? Defence without specialized immune cells. Nat Rev Immunol 12:89-100

Takagi H, Abe A, Yoshida K, Kosugi S, Natsume S, Mitsuoka C et al (2013) QTL-seq: rapid mapping of quantitative trait loci in rice by whole genome resequencing of DNA from two bulked populations. Plant J 74:174-183

Van Ooijen JW (2006) JoinMap 4, software for the calculation of genetic linkage maps in experimental populations. Kyazma B.V, Wageningen

van Ooijen G, Mayr G, Kasiem MM, Albrecht M, Cornelissen BJ, Takken FL (2008) Structure-function analysis of the NB-ARC domain of plant disease resistance proteins. J Exp Bot 59:1383-1397

Varshney RK, Bertioli DJ, Moretzsohn MC, Vadez V, Krishnamurthy L, Aruna R et al (2009) The first SSR-based genetic linkage map for cultivated groundnut (Arachis hypogaea L.). Theor Appl Genet 118:729-739

Varshney RK, Mohan SM, Gaur PM, Gangarao NV, Pandey MK, Bohra A et al (2013) Achievements and prospects of genomics-assisted breeding in three legume crops of the semi-arid tropics. Biotechnol Adv 31:1120-1134

Varshney RK, Pandey MK, Janila P, Nigam SN, Sudini H, Gowda MV et al (2014) Marker-assisted introgression of a QTL region to improve rust resistance in three elite and popular varieties of peanut (Arachis hypogaea L.). Theor Appl Genet 127:1771-1781

Vishwakarma MK, Nayak SN, Guo B, Wan L, Liao B, Varshney RK, Pandey MK (2017) Classical and molecular approaches for mapping of genes and quantitative trait loci in peanut. In: Varshney RK, Pandey MK, Puppala N (eds) The peanut genome. Springer, Cham, pp 93-116. https://doi.org/10.1007/978-3-319-63935-2_7

Voorrips RE (2002) MapChart: software for the graphical presentation of linkage maps and QTLs. J Hered 93:77-78
Wambugu P, Ndjiondjop MN, Furtado A, Henry R (2018) Sequencing of bulks of segregants allows dissection of genetic control of amylose content in rice. Plant Biotechnol J 16:100-110

Wang S, Basten CJ, Zeng Z-B (2012) Windows QTL Cartographer 2.5. Department of Statistics, North Carolina State University, Raleigh. https://statgen.ncsu.edu/qtlcart/WQTLCart.htm. Accessed 01 Dec 2015

Wang L, Zhou X, Ren X, Huang L, Luo H, Chen Y et al (2018a) A major and stable QTL for bacterial wilt resistance on chromosome B02 identified using a high-density SNP-based genetic linkage map in cultivated peanut Yuanza 9102 derived population. Front Genet 9:652

Wang Z, Huai D, Zhang Z, Cheng K, Kang Y, Wan L et al (2018b) Development of a high-density genetic map based on specific length amplified fragment sequencing and its application in quantitative trait loci analysis for yield-related traits in cultivated peanut. Front Plant Sci 9:827

Wu G, Liu S, Zhao Y, Wang W, Kong Z, Tang D (2015) ENHANCED DISEASE RESISTANCE4 associates with CLATHRIN HEAVY CHAIN2 and modulates plant immunity by regulating relocation of EDR1 in Arabidopsis. Plant Cell 27:857-873

Yu B, Huai D, Huang L, Kang Y, Ren X, Chen Y et al (2019) Identification of genomic regions and diagnostic markers for resistance to aflatoxin contamination in peanut (Arachis hypogaea $\mathrm{L}$.). BMC Genet 20:32

Zhao Y, Zhang C, Chen H, Yuan M, Nipper R, Prakash CS et al (2016) QTL mapping for bacterial wilt resistance in peanut (Arachis hypogaea L.). Mol Breed 36:13

Zhong C, Sun SL, Li YP, Duan CX, Zhu ZD (2018) Next-generation sequencing to identify candidate genes and develop diagnostic markers for a novel Phytophthora resistance gene, RpsHC18, in soybean. Theor Appl Genet 131:525-538

Zhou J, Loh YT, Bressan RA, Martin GB (1995) The tomato gene Ptil encodes a serine/threonine kinase that is phosphorylated by Pto and is involved in the hypersensitive response. Cell 83:925-935

Zhou X, Xia Y, Ren X, Chen Y, Huang L, Huang S et al (2014) Construction of a SNP-based genetic linkage map in cultivated peanut based on large scale marker development using next-generation double-digest restriction-site-associated DNA sequencing (ddRADseq). BMC Genom 15:14

Zhuang W, Chen H, Yang M, Wang J, Pandey MK, Zhang C et al (2019) The genome of cultivated peanut provides insight into legume karyotypes, polyploid evolution and crop domestication. Nat Genet 51:865-876

Publisher's Note Springer Nature remains neutral with regard to jurisdictional claims in published maps and institutional affiliations. 